\title{
A Comparative Study of Dosimetric Analysis of Three Different Sets of Five Fields and Seven Fields IMRT Plans for Prostate Cancer
}

\author{
Mukesh Kumar Zope', Deepali Bhaskar Patil'2, Angel Kuriakose ${ }^{3}$, Aslam Rahman ${ }^{3}$, \\ Vinita Trivedi' ${ }^{3}$, Shekhar Kumar Keshri²

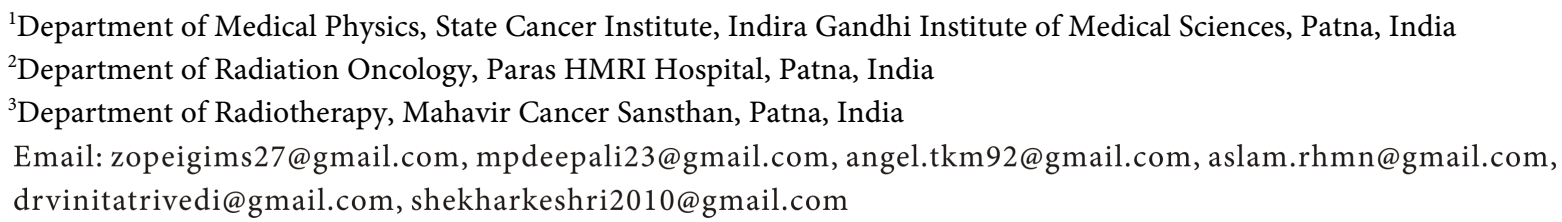

How to cite this paper: Zope, M.K., Patil, D.B., Kuriakose, A., Rahman, A., Trivedi, V. and Keshri, S.K. (2019) A Comparative Study of Dosimetric Analysis of Three Different Sets of Five Fields and Seven Fields IMRT Plans for Prostate Cancer. International Journal of Medical Physics, Clinical Engineering and Radiation Oncology, 8, 175-192.

https://doi.org/10.4236/ijmpcero.2019.83016

Received: July 7, 2019

Accepted: August 26, 2019

Published: August 29, 2019

Copyright $\odot 2019$ by author(s) and Scientific Research Publishing Inc. This work is licensed under the Creative Commons Attribution International License (CC BY 4.0).

http://creativecommons.org/licenses/by/4.0/

\begin{abstract}
The advent of Intensity Modulated Radiation Therapy (IMRT) is rapidly changing the field of Radiation Oncology. IMRT has the potential to improve clinical implementation of highly conformal non-convex dose distributions. Nonetheless, a number of IMRT approaches including coplanar and noncoplanar beam techniques with 5 to 9 beams at different angles have been used in an effort to get the best dose distribution. The purpose of this study is to compare the dose to normal tissue and dose-limiting structures, conformity index, homogeneity index, number of monitor units required for the treatment of prostate cancer in 3 sets of five and seven beam IMRT plans and thus to select the best IMRT approach for patients with prostate cancer.
\end{abstract}

\section{Keywords}

Prostate Cancer, MUs, Conformity Index, Homogeneity Index

\section{Introduction}

Intensity-Modulated Radiation Therapy (IMRT) is significant Radiation delivery technique for prostate cancer. However, the anatomical location of the prostate, which is in close proximity to the rectum, bladder, penile bulb, and femoral heads exposes these organs at risk of receiving radiation dose and subsequent toxicity during prostate cancer treatment by radiation. Historically, radiation was delivered using 4 field box techniques, which limited the total dose that could be delivered to the prostate without exceeding the tolerance dose limit of 
surrounding critical normal structures. Multiple studies have shown an improved tumor control rate with dose-escalated radiation therapy to prostate [1] [2] [3] [4] several single institution series has reported a reduction in late toxicity with the introduction of IMRT compared to 3DCRT, even with dose escalation [4] [5].

The use of IMRT in the treatment of localized prostate cancer is associated with significantly lower doses of radiation delivered to the rectum and bladder. The ability of IMRT to reduce dose to nearby normal structures without sacrificing dose coverage of the target makes it an ideal treatment modality for prostate cancer.

The beam orientations used for the IMRT treatment of prostate cancer are still selected empirically, without any guideline. In the past several groups have investigated the number and orientations of beams in IMRT of prostate cancer [6] [7]. Practically in the treatment of prostate cancer by IMRT technique five to seven beams of different gantry angle are used.

The aim of this study is to compare the dose to normal tissues and dose-limiting structures such as rectum and urinary bladder, conformity index, homogeneity index, number of monitor units required for the treatment of prostate cancer when using the same prescription doses, same planning system and PTV margins with 3 sets of five and seven beam IMRT plans.

\section{Methods \& Material}

A thermoplastic pelvic mask was used to immobilize the patients and CT reference isocenter were marked on the mask with the help of fidiucial lead balls. Planning CT scan with $3 \mathrm{~mm}$ thickness was acquired in the treatment position from level L2-3 to the ischial tuberosity. They were placed in supine position with special instruction to keep their rectum empty and bladder comfortably full at the time of simulation and during each treatment fraction. CT slices of patient were transferred to Elekta focal-SIM contouring workstation via DICOM for contouring.

For this study, 6 different IMRT plans were created for each of the 10 patients undergoing radical radiation treatment for prostate cancer. The IMRT plans generated included 3 different set of 5 beam gantry angles $5 \mathrm{~B} 1\left(0^{\circ}, 50^{\circ}, 100^{\circ}\right.$, $\left.260^{\circ}, 310^{\circ}\right)$ as shown in Figure $1,5 \mathrm{~B} 2\left(45^{\circ}, 105^{\circ}, 180^{\circ}, 225^{\circ}, 315^{\circ}\right)$ as shown in Figure 2, $5 \mathrm{~B} 3\left(0^{\circ}, 75^{\circ}, 135^{\circ}, 225^{\circ}, 285^{\circ}\right)$ as shown in Figure 3 and 3 different sets of 7 beam gantry angle $7 \mathrm{~B} 1\left(0^{\circ}, 51^{\circ}, 102^{\circ}, 153^{\circ}, 204^{\circ}, 255^{\circ}, 306^{\circ}\right)$ as shown in Figure $4,7 \mathrm{~B} 2\left(0^{\circ}, 37^{\circ}, 75^{\circ}, 135^{\circ}, 225^{\circ}, 285^{\circ}, 327^{\circ}\right)$ as shown in Figure $5,7 \mathrm{~B} 3$ $\left(25^{\circ}, 75^{\circ}, 130^{\circ}, 180^{\circ}, 230^{\circ}, 280^{\circ}, 335^{\circ}\right)$ as shown in Figure 6. Thus, a total of 60 plans were generated using step \& shoot treatment delivery technique with 80 leaf Multileaf collimator and leaf width of $1 \mathrm{~cm}$ at the isocenter. The IMRT plans were created using Xio TPS with $6 \mathrm{MV}$ photon and superposition algorithms. A hypo fractionated prescription dose of $70 \mathrm{~Gy} / 28 \#$ at 2.5 Gy per fraction was used. The plans were evaluated with the help of Dose Volume Histogram. 


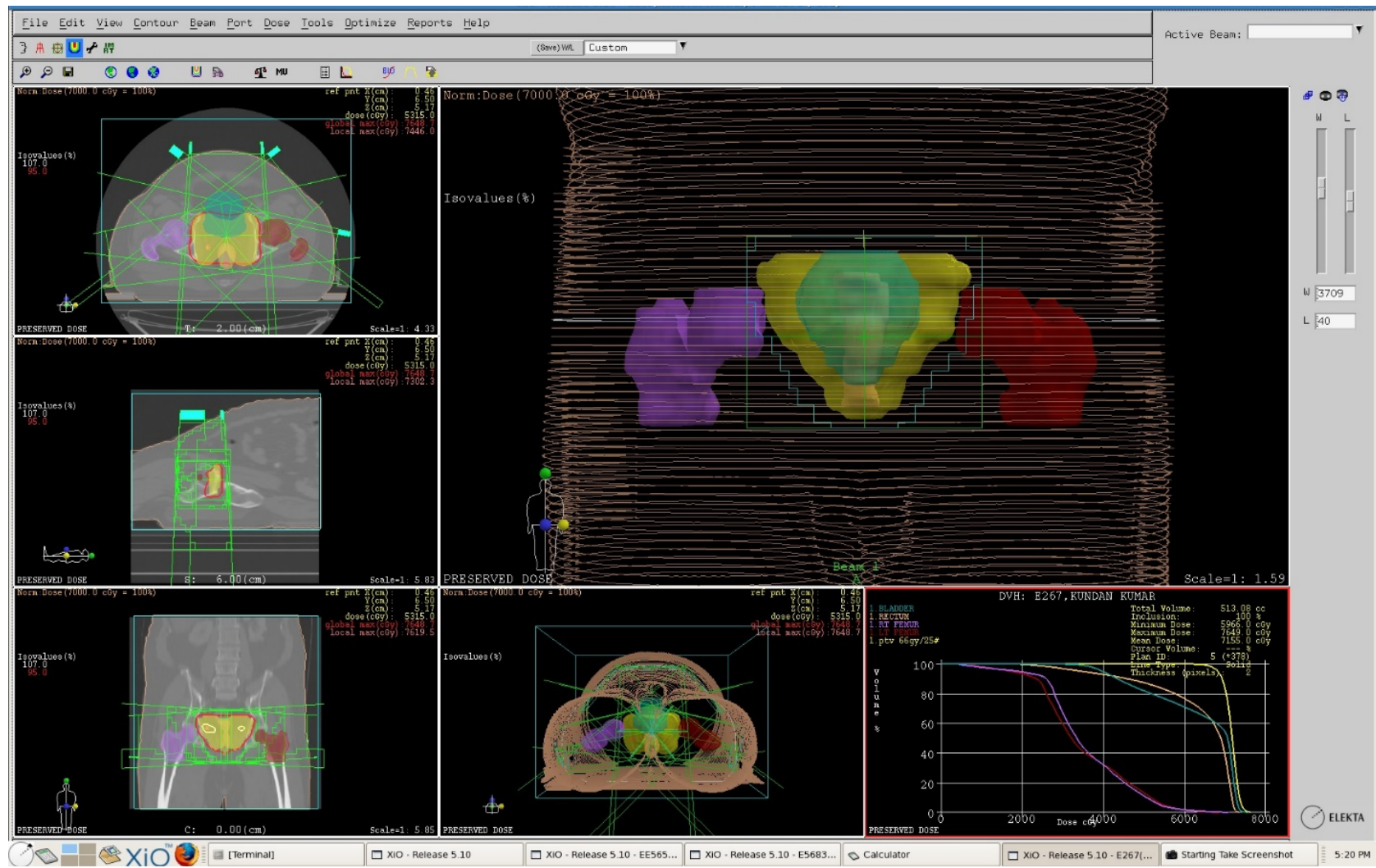

Figure 1. 5B1 $\left(0^{\circ}, 50^{\circ}, 100^{\circ}, 260^{\circ}, 310^{\circ}\right)$.

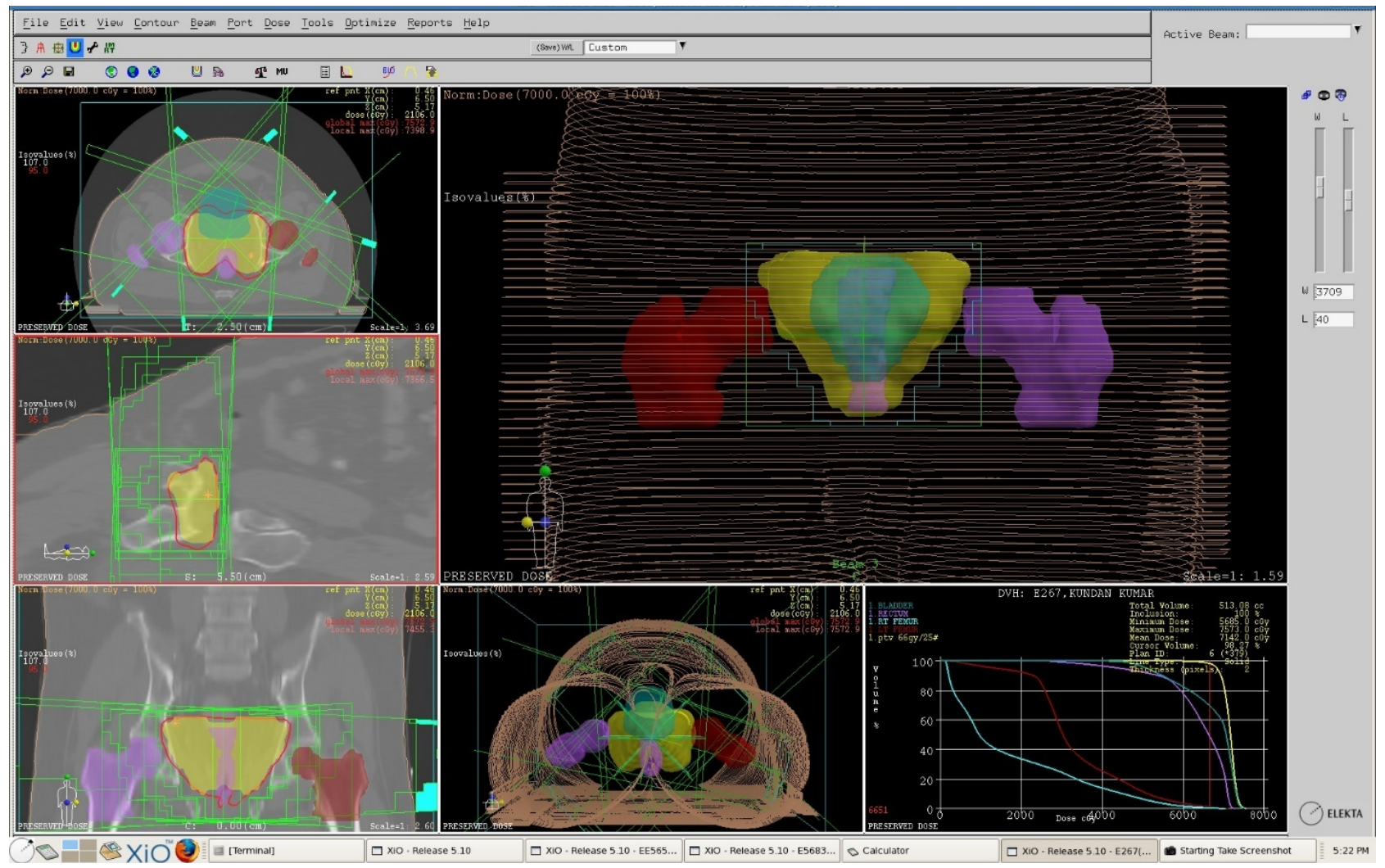

Figure $2.5 \mathrm{~B} 2\left(45^{\circ}, 105^{\circ}, 180^{\circ}, 225^{\circ}, 315^{\circ}\right)$. 


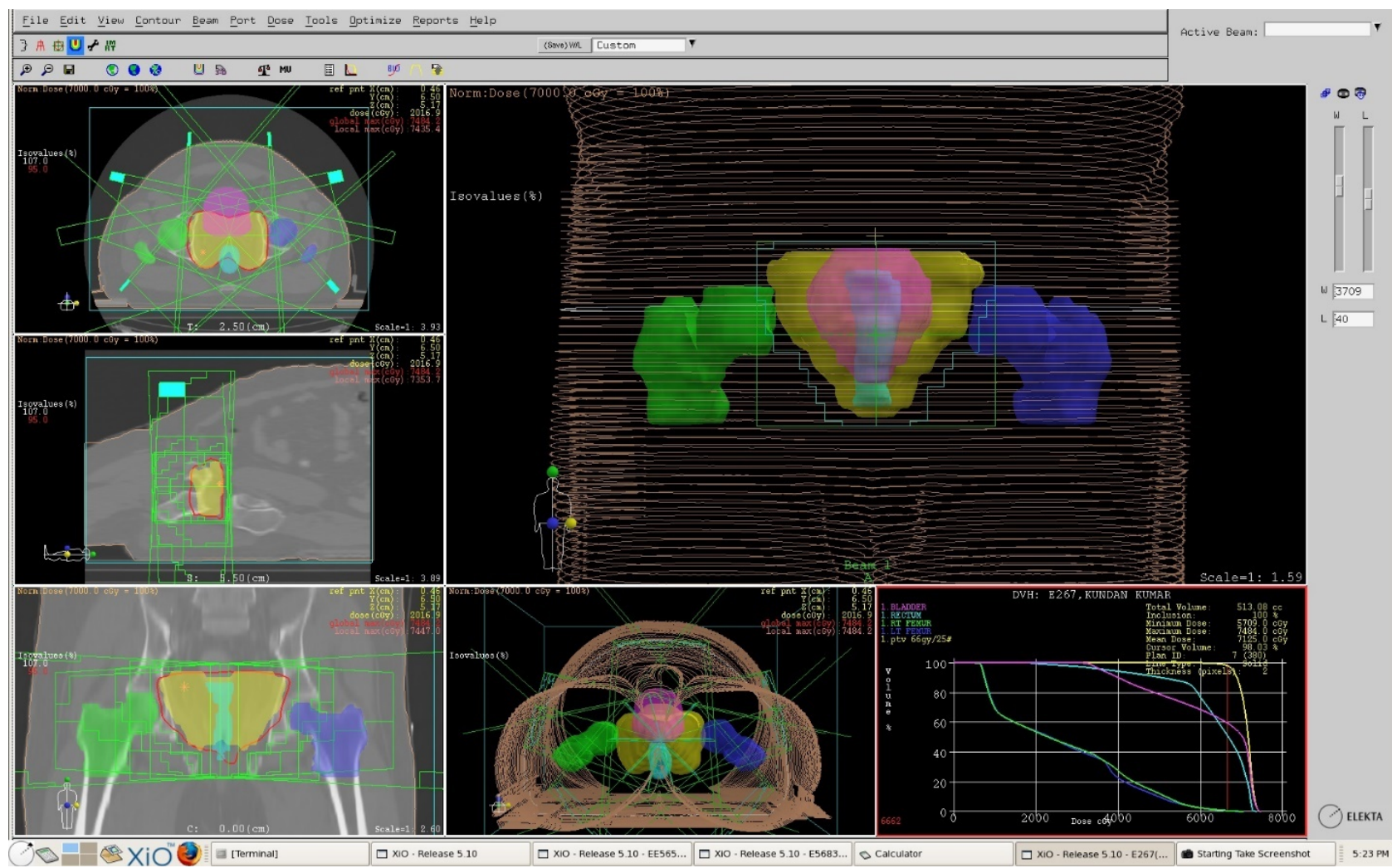

Figure 3. $5 \mathrm{~B} 3\left(0^{\circ}, 75^{\circ}, 135^{\circ}, 225^{\circ}, 285^{\circ}\right)$.

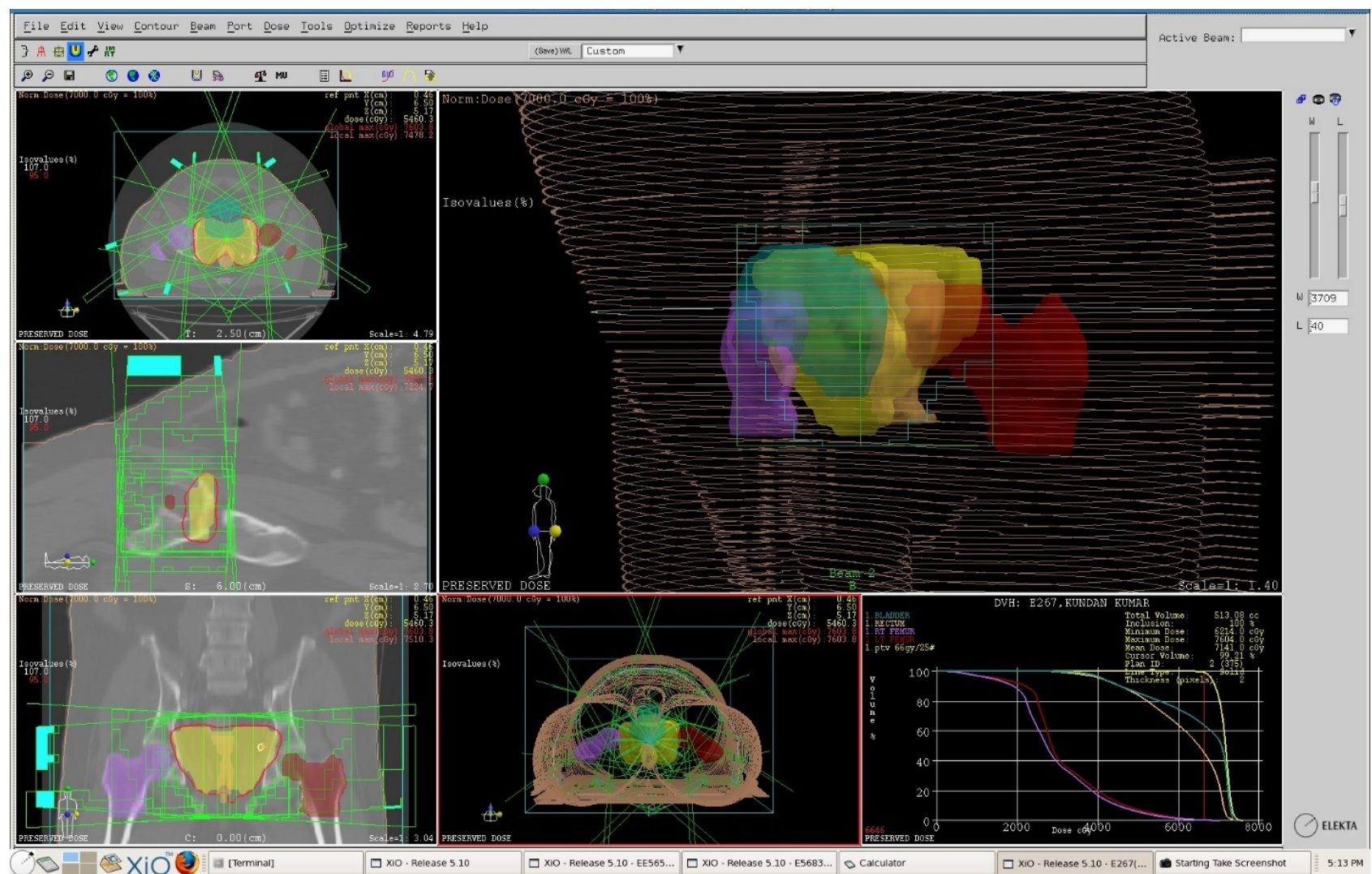

Figure 4. $7 \mathrm{~B} 1\left(0^{\circ}, 51^{\circ}, 102^{\circ}, 153^{\circ}, 204^{\circ}, 255^{\circ}, 306^{\circ}\right)$. 


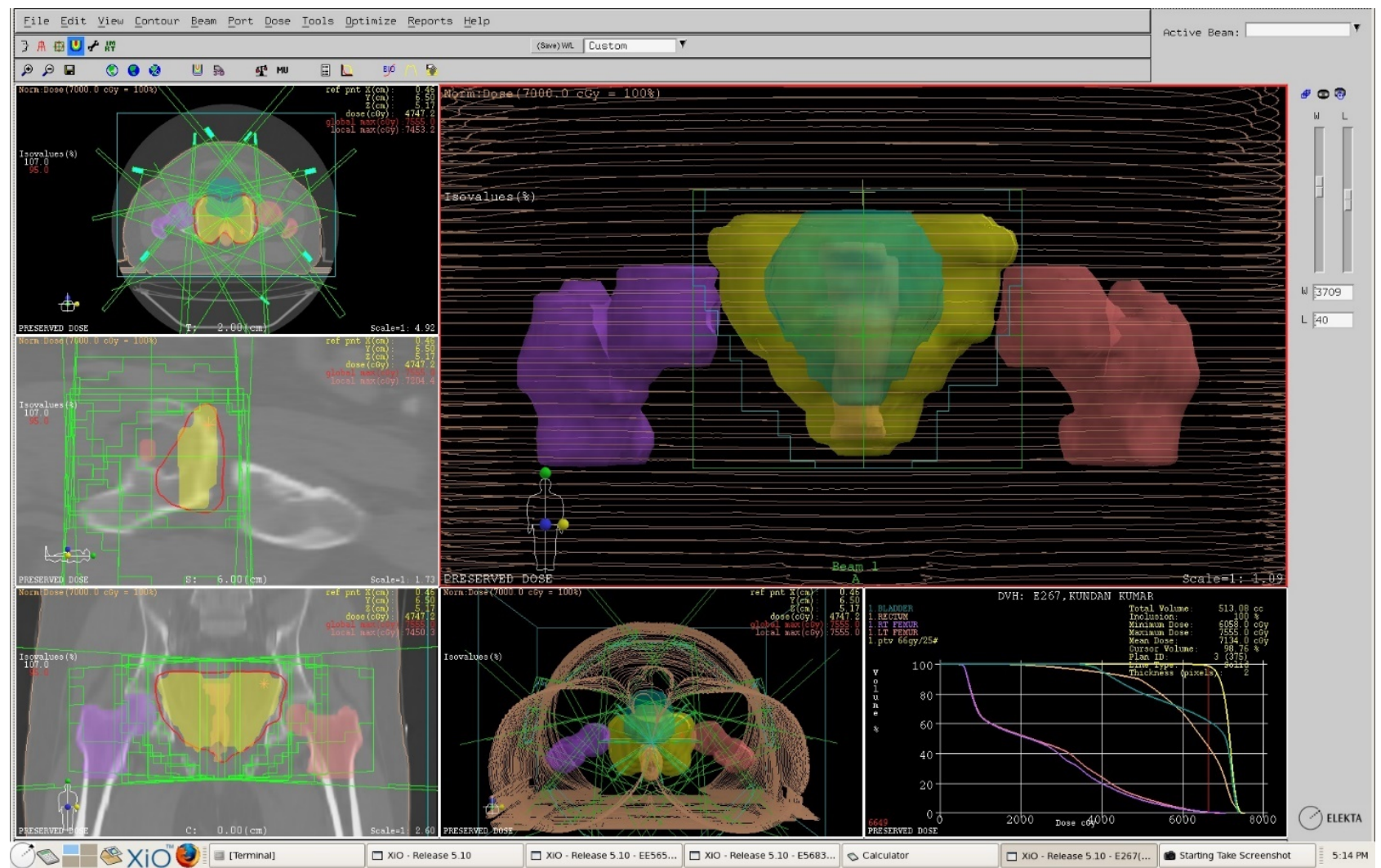

Figure 5. $7 \mathrm{~B} 2\left(0^{\circ}, 37^{\circ}, 75^{\circ} 135^{\circ}, 225^{\circ}, 285^{\circ}, 327^{\circ}\right)$.

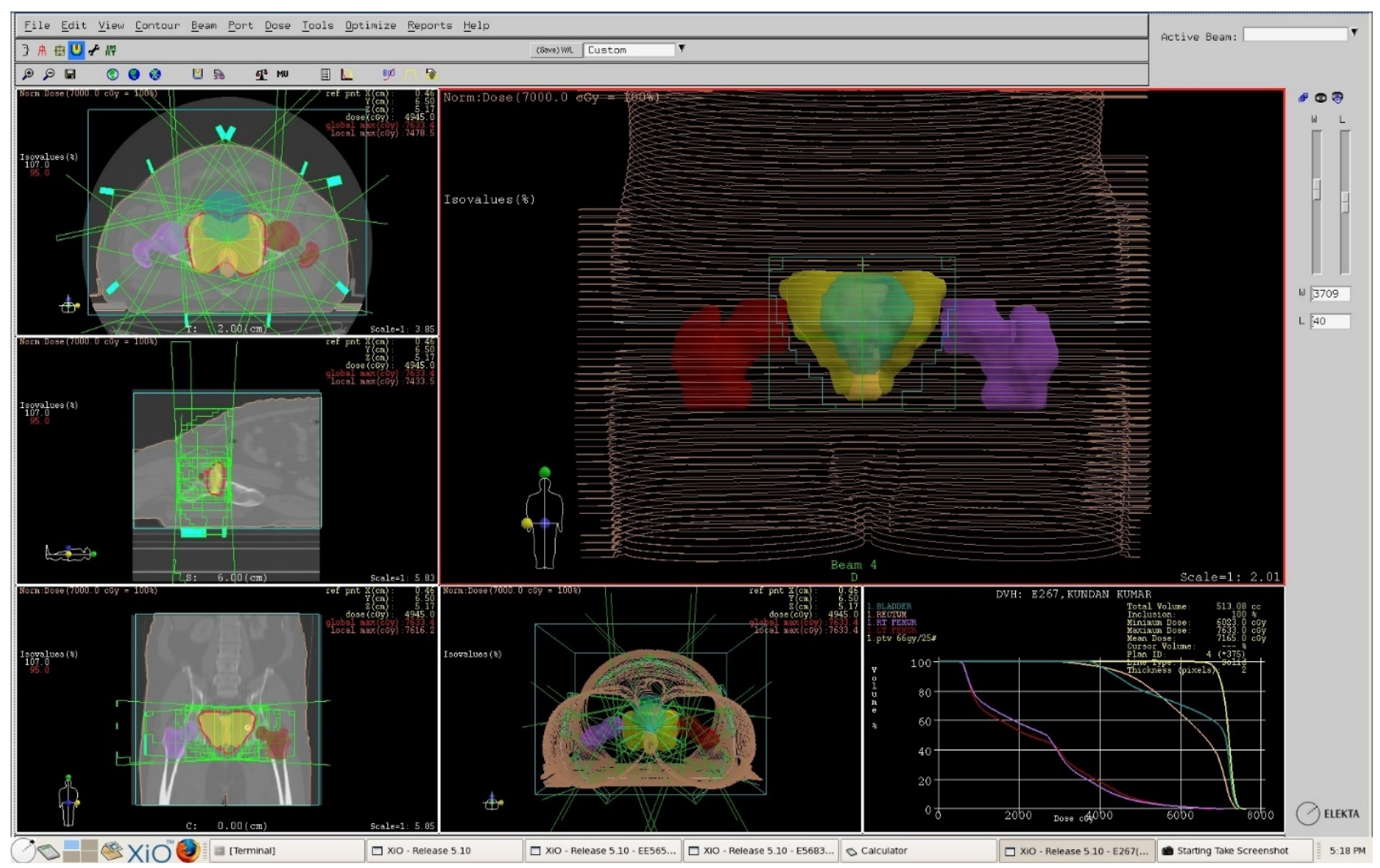

Figure 6. $7 \mathrm{~B} 3\left(25^{\circ}, 75^{\circ}, 130^{\circ}, 180^{\circ}, 230^{\circ}, 280^{\circ}, 335^{\circ}\right)$. 
The dose coverage for each patient's six different IMRT plans for the PTV are designated to an international Commission Radiation Units and Measurements report 62 reference point in the PTV, Medium coverage of the planned target volume to be $95 \%$ of the prescribed dose while the maximum dose in the target volume to be not greater than $107 \%$ of the prescribed dose and number of MUs were evaluated. Normal tissue like rectum and urinary bladder receiving maximum, Minimum and V65 Gy $<35 \%$ \& V70 Gy $<25 \%$ volume in different IMRT plans are calculated. Femoral head maximum dose is below 54 Gy and Penile bulb $\mathrm{D}_{\text {mean }}$ less than or equal to $52.5 \mathrm{~Gy}$ (optional in the protocol) for all plans.

Analysis of Physical Parameter in Intensity Modulated Radiotherapy Plans:

The plans were evaluated for approval with following Plan evaluation parameters:

- $\mathrm{HI}-$ Homogeneity Index $=\mathrm{D} 5 \% / \mathrm{D} 95 \%$, where $\mathrm{D} 5 \%$ and $\mathrm{D} 95 \%$ are minimum dose to $5 \%$ \& $95 \%$ of the target volume respectively [8].

- CI formula as suggested by ICRU Report 62 which was originally reported in RTOG 90-05 protocol [9]. CI-Conformity Index (for 95\% of PD) = Volume receiving 95\% of PD/PTV

Homogeneity index indicates the dose homogeneity in the target volume and Conformity index indicates the dose confinement near to target volume and level of dose spillage in normal tissue volume around the target volume. Value of $\mathrm{HI}$ and $\mathrm{CI}=1$ is best; although it is not possible practically and the value of both indices always remain higher than 1 lower than near about 1 .

\section{Statistical Analysis}

Data was entered on excel sheet and ANOVA with turkey multiple comparison test and Kruskal-Wallis test with Dunn's Multiple Comparison Test was applied for parametric and non-parametric data respectively using Graph pad (version 5) software. A P-value $\leq 0.05$ was considered statistically significant.

\section{Result}

The doses which are delivered to the V65 \& V70 Urinary Bladder dose in different IMRT plans are as shown in Tables 1-4 and we observed non-significant $(\mathrm{P}>0.05)$ difference between the groups using ANOVA test with turkey multiple comparison test.

The doses which are delivered to the V65 \& V70 rectum dose in different IMRT plans are as shown in Tables 5-8 and we observed non-significant $(\mathrm{P}>$ 0.05) difference between the groups using Kruskal-Wallis test with Dunn's Multiple Comparison Test.

Mean of Maximum doses deliver to bladder in all 6 IMRT plans 5B1, 5B2, 5B3, 7B1, 7B2, 7B3 to all 10 patients are $75.29 \mathrm{~Gy}, 74.99 \mathrm{~Gy}, 74.60 \mathrm{~Gy}, 74.98 \mathrm{~Gy}$, 75.25 Gy and 75.22 Gy $(\mathrm{P}=0.0 .00023,0.787912 / \mathrm{NS},=0.000995,0.000113$ and 0.000342). But there is non-significant difference between all IMRT accept 5B3 
Table 1. V65 Urinary bladder dose in different IMRT plans.

\begin{tabular}{|c|c|c|}
\hline \multicolumn{2}{|c|}{ V65 Bladder } & \multirow{2}{*}{ ANOVA test (P value) } \\
\hline Group & Mean \pm SD & \\
\hline $7 \mathrm{~B} 1$ & $38.28 \pm 15.49$ & \multirow{6}{*}{0.9678} \\
\hline 7B2 & $39.92 \pm 18.86$ & \\
\hline 7B3 & $38.34 \pm 15.36$ & \\
\hline $5 \mathrm{~B} 1$ & $38.80 \pm 4.70$ & \\
\hline $5 \mathrm{~B} 2$ & $44.01 \pm 16.32$ & \\
\hline $5 \mathrm{~B} 3$ & $39.68 \pm 14.46$ & \\
\hline
\end{tabular}

Table 2. Tukey's multiple comparison test for V65 of urinary bladder in different IMRT plans.

\begin{tabular}{ll}
\hline Tukey's Multiple Comparison Test & Summary \\
\hline $7 \mathrm{~B} 1$ vs $7 \mathrm{~B} 2$ & $\mathrm{~ns}$ \\
$7 \mathrm{~B} 1$ vs $7 \mathrm{~B} 3$ & $\mathrm{~ns}$ \\
$7 \mathrm{~B} 1$ vs $5 \mathrm{~B} 1$ & $\mathrm{~ns}$ \\
$7 \mathrm{~B} 1$ vs $5 \mathrm{~B} 2$ & $\mathrm{~ns}$ \\
$7 \mathrm{~B} 1$ vs $5 \mathrm{~B} 3$ & $\mathrm{~ns}$ \\
$7 \mathrm{~B} 2$ vs $7 \mathrm{~B} 3$ & $\mathrm{~ns}$ \\
$7 \mathrm{~B} 2$ vs $5 \mathrm{~B} 1$ & $\mathrm{~ns}$ \\
$7 \mathrm{~B} 2$ vs $5 \mathrm{~B} 2$ & $\mathrm{~ns}$ \\
$7 \mathrm{~B} 2$ vs $5 \mathrm{~B} 3$ & $\mathrm{~ns}$ \\
$7 \mathrm{~B} 3$ vs $5 \mathrm{~B} 1$ & $\mathrm{~ns}$ \\
$7 \mathrm{~B} 3$ vs $5 \mathrm{~B} 2$ & $\mathrm{~ns}$ \\
$7 \mathrm{~B} 3$ vs $5 \mathrm{~B} 3$ & $\mathrm{~ns}$ \\
$5 \mathrm{~B} 1$ vs $5 \mathrm{~B} 2$ & $\mathrm{~ns}$ \\
$5 \mathrm{~B} 1$ vs $5 \mathrm{~B} 3$ & $\mathrm{~ns}$ \\
$5 \mathrm{~B} 2$ vs $5 \mathrm{~B} 3$ & \\
\hline
\end{tabular}

Significant (s), Non-Significant (ns).

Table 3. V70 urinary bladder dose in different IMRT plans.

\begin{tabular}{ccc}
\hline \multicolumn{2}{c}{ V70 Bladder } & ANOVA test (P value) \\
\hline Group & Mean $\pm \mathrm{SD}$ & \\
\hline $7 \mathrm{~B} 1$ & $25.40 \pm 13.27$ & \\
$7 \mathrm{~B} 2$ & $25.11 \pm 14.42$ & 0.999 \\
$7 \mathrm{~B} 3$ & $26.55 \pm 14.34$ & \\
$5 \mathrm{~B} 1$ & $26.33 \pm 13.37$ & \\
$5 \mathrm{~B} 2$ & $25.95 \pm 14.07$ & \\
$5 \mathrm{~B} 3$ & $25.84 \pm 13.67$ & \\
\hline
\end{tabular}


Table 4. Tukey's multiple comparison test for V70 urinary bladder dose in different IMRT plans.

\begin{tabular}{lc}
\hline Tukey's Multiple Comparison Test & Summary \\
\hline $7 \mathrm{~B} 1$ vs $7 \mathrm{~B} 2$ & $\mathrm{~ns}$ \\
$7 \mathrm{~B} 1$ vs $7 \mathrm{~B} 3$ & $\mathrm{~ns}$ \\
$7 \mathrm{~B} 1$ vs $5 \mathrm{~B} 1$ & $\mathrm{~ns}$ \\
$7 \mathrm{~B} 1$ vs $5 \mathrm{~B} 2$ & $\mathrm{~ns}$ \\
$7 \mathrm{~B} 1$ vs $5 \mathrm{~B} 3$ & $\mathrm{~ns}$ \\
$7 \mathrm{~B} 2$ vs $7 \mathrm{~B} 3$ & $\mathrm{~ns}$ \\
$7 \mathrm{~B} 2$ vs $5 \mathrm{~B} 1$ & $\mathrm{~ns}$ \\
$7 \mathrm{~B} 2$ vs $5 \mathrm{~B} 2$ & $\mathrm{~ns}$ \\
$7 \mathrm{~B} 2$ vs $5 \mathrm{~B} 3$ & $\mathrm{~ns}$ \\
$7 \mathrm{~B} 3$ vs $5 \mathrm{~B} 1$ & $\mathrm{~ns}$ \\
$7 \mathrm{~B} 3$ vs $5 \mathrm{~B} 2$ & $\mathrm{~ns}$ \\
$7 \mathrm{~B} 3$ vs $5 \mathrm{~B} 3$ & $\mathrm{~ns}$ \\
$5 \mathrm{~B} 1$ vs $5 \mathrm{~B} 2$ & $\mathrm{~ns}$ \\
$5 \mathrm{~B} 1$ vs $5 \mathrm{~B} 3$ & $\mathrm{~ns}$ \\
$5 \mathrm{~B} 2$ vs $5 \mathrm{~B} 3$ &
\end{tabular}

Significant (s), Non-Significant (ns).

Table 5. V65 rectum dose in different IMRT plans.

\begin{tabular}{|c|c|c|}
\hline \multicolumn{2}{|c|}{ V65 Rectum } & \multirow{2}{*}{ ANOVA test (P value) } \\
\hline Group & Mean \pm SD & \\
\hline 7B1 & $34.99 \pm 16.08$ & \multirow{7}{*}{0.8050} \\
\hline 7B2 & $36.03 \pm 17.16$ & \\
\hline $7 \mathrm{~B} 3$ & $35.59 \pm 17.36$ & \\
\hline & & \\
\hline $5 \mathrm{~B} 1$ & $41.88 \pm 19.21$ & \\
\hline $5 \mathrm{~B} 2$ & $40.42 \pm 17.35$ & \\
\hline $5 \mathrm{~B} 3$ & $44.64 \pm 23.05$ & \\
\hline
\end{tabular}

as shown in Figure 7 \& Figure 8.

Mean of Maximum doses deliver to rectum in all 6 IMRT plans 5B1, 5B2, 5B3, 7B1, 7B2, and 7B3 to all 10 patients are 73.78 Gy, 73.36 Gy, 74.21 Gy, 74.50 Gy, 74.34 Gy and 74.21 Gy, ( $\mathrm{P}=0.35951 / \mathrm{NS}, 0.560054 / \mathrm{NS}, 0.026766 / \mathrm{S}, \mathrm{P}=0.018904 / \mathrm{S}$, $0.027704 / \mathrm{S}$ and 0 respectively). But there is significant difference between all IMRT accept 5B3 as shown in Figure 9 \& Figure 10.

For conformity index of various IMRT plans there is non-significant $(\mathrm{P}>$ 0.05) difference between the various groups using ANOVA test with turkey 
Table 6. Tukey's multiple comparison test for V65 rectum dose in different IMRT plans.

\begin{tabular}{cc}
\hline Tukey's Multiple Comparison Test & Summary \\
\hline $7 \mathrm{~B} 1$ vs $7 \mathrm{~B} 2$ & $\mathrm{~ns}$ \\
$7 \mathrm{~B} 1$ vs $7 \mathrm{~B} 3$ & $\mathrm{~ns}$ \\
$7 \mathrm{~B} 1$ vs $5 \mathrm{~B} 1$ & $\mathrm{~ns}$ \\
$7 \mathrm{~B} 1$ vs $5 \mathrm{~B} 2$ & $\mathrm{~ns}$ \\
$7 \mathrm{~B} 1$ vs $5 \mathrm{~B} 3$ & $\mathrm{~ns}$ \\
$7 \mathrm{~B} 2$ vs $7 \mathrm{~B} 3$ & $\mathrm{~ns}$ \\
$7 \mathrm{~B} 2$ vs $5 \mathrm{~B} 1$ & $\mathrm{~ns}$ \\
$7 \mathrm{~B} 2$ vs $5 \mathrm{~B} 2$ & $\mathrm{~ns}$ \\
$7 \mathrm{~B} 2$ vs $5 \mathrm{~B} 3$ & $\mathrm{~ns}$ \\
$7 \mathrm{~B} 3$ vs $5 \mathrm{~B} 1$ & $\mathrm{~ns}$ \\
$7 \mathrm{~B} 3$ vs $5 \mathrm{~B} 2$ & $\mathrm{~ns}$ \\
$7 \mathrm{~B} 3$ vs $5 \mathrm{~B} 3$ & $\mathrm{~ns}$ \\
$5 \mathrm{~B} 1$ vs $5 \mathrm{~B} 2$ & $\mathrm{~ns}$ \\
$5 \mathrm{~B} 1$ vs $5 \mathrm{~B} 3$ & $\mathrm{~ns}$ \\
$5 \mathrm{~B} 2$ vs $5 \mathrm{~B} 3$ & \\
\hline
\end{tabular}

Significant (s), Non-Significant (ns).

Table 7. V70 rectum dose in different IMRT plans.

\begin{tabular}{ccc}
\hline \multicolumn{2}{c}{ V70 Rectum } & ANOVA test (P value) \\
\hline Group & Mean $\pm \mathrm{SD}$ & \\
\hline 7B1 & $18.55 \pm 11.64$ & 0.8996 \\
$7 \mathrm{~B} 2$ & $17.95 \pm 11.83$ & \\
$7 \mathrm{~B} 3$ & $18.90 \pm 12.07$ & \\
5B1 & $19.35 \pm 12.52$ & \\
5B2 & $17.88 \pm 11.17$ & \\
5B3 & $23.92 \pm 16.46$ & \\
\hline
\end{tabular}

multiple comparison test as shown in Figure 11 and Table 9 and Table 10.

For Homogeneity index of various IMRT plans there are non-significant $(\mathrm{P}>$ 0.05) difference between the various groups using ANOVA test with turkey multiple comparison test as shown in Figure 12 and as shown in Table 11 and Table 12 .

For Monitor Unit of various IMRT plans there are non-significant $(\mathrm{P}>0.05)$ difference between the groups using Kruskal-Wallis test with Dunn's Multiple Comparison Test as shown in Table 13 \& Table 14.

5 field IMRT plans achieved a $14.9 \%$ relative decrease in the mean number of monitor units for radiation delivery as compared to 7 field IMRT plans (mean 
Table 8. Tukey's multiple comparison test for V70 rectum dose in different IMRT plans.

\begin{tabular}{cc}
\hline Tukey's Multiple Comparison Test & Summary \\
\hline $7 \mathrm{~B} 1$ vs $7 \mathrm{~B} 2$ & $\mathrm{~ns}$ \\
$7 \mathrm{~B} 1$ vs $7 \mathrm{~B} 3$ & $\mathrm{~ns}$ \\
$7 \mathrm{~B} 1$ vs $5 \mathrm{~B} 1$ & $\mathrm{~ns}$ \\
$7 \mathrm{~B} 1$ vs $5 \mathrm{~B} 2$ & $\mathrm{~ns}$ \\
$7 \mathrm{~B} 1$ vs $5 \mathrm{~B} 3$ & $\mathrm{~ns}$ \\
$7 \mathrm{~B} 2$ vs $7 \mathrm{~B} 3$ & $\mathrm{~ns}$ \\
$7 \mathrm{~B} 2$ vs $5 \mathrm{~B} 1$ & $\mathrm{~ns}$ \\
$7 \mathrm{~B} 2$ vs $5 \mathrm{~B} 2$ & $\mathrm{~ns}$ \\
$7 \mathrm{~B} 2$ vs $5 \mathrm{~B} 3$ & $\mathrm{~ns}$ \\
$7 \mathrm{~B} 3$ vs $5 \mathrm{~B} 1$ & $\mathrm{~ns}$ \\
$7 \mathrm{~B} 3$ vs $5 \mathrm{~B} 2$ & $\mathrm{~ns}$ \\
$7 \mathrm{~B} 3$ vs $5 \mathrm{~B} 3$ & $\mathrm{~ns}$ \\
$5 \mathrm{~B} 1$ vs $5 \mathrm{~B} 2$ & $\mathrm{~ns}$ \\
$5 \mathrm{~B} 1$ vs $5 \mathrm{~B} 3$ & $\mathrm{~ns}$ \\
$5 \mathrm{~B} 2$ vs $5 \mathrm{~B} 3$ & \\
\hline
\end{tabular}

Significant (s), Non-Significant (ns).

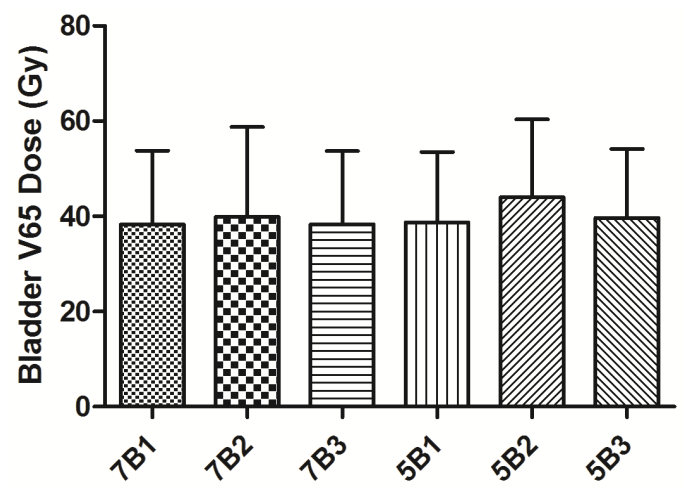

Figure 7. V65 urinary bladder dose in different IMRT plans.

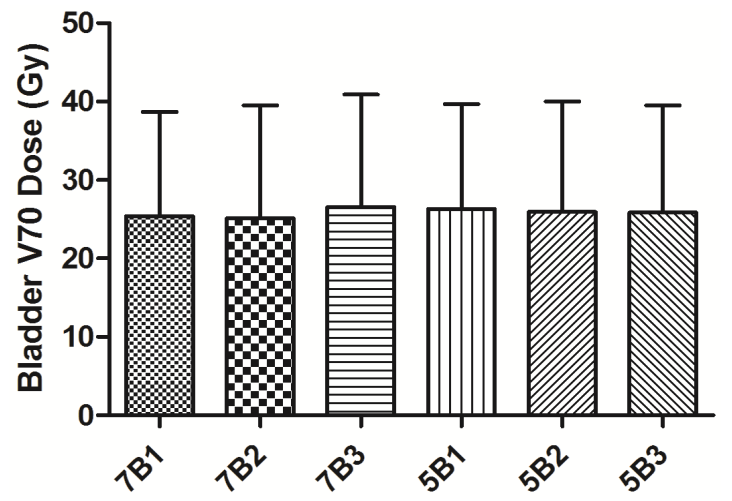

Figure 8. V70 urinary bladder dose in different IMRT plans. 


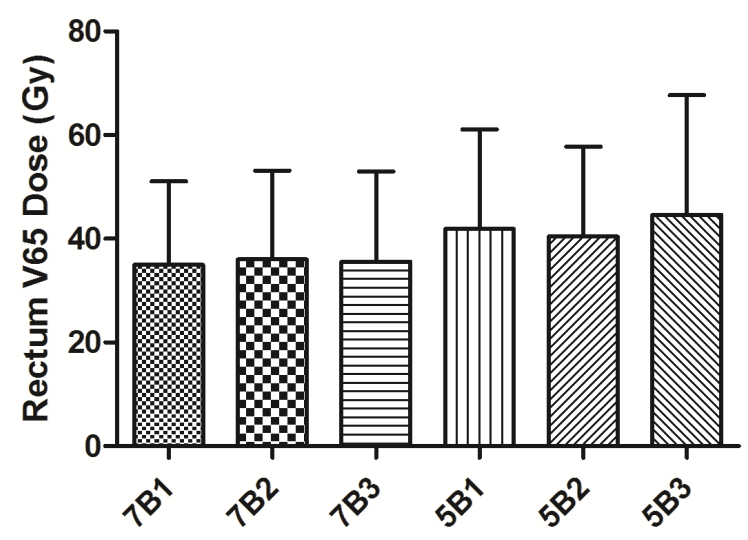

Figure 9. Rectum V65 dose for different IMRT plans.

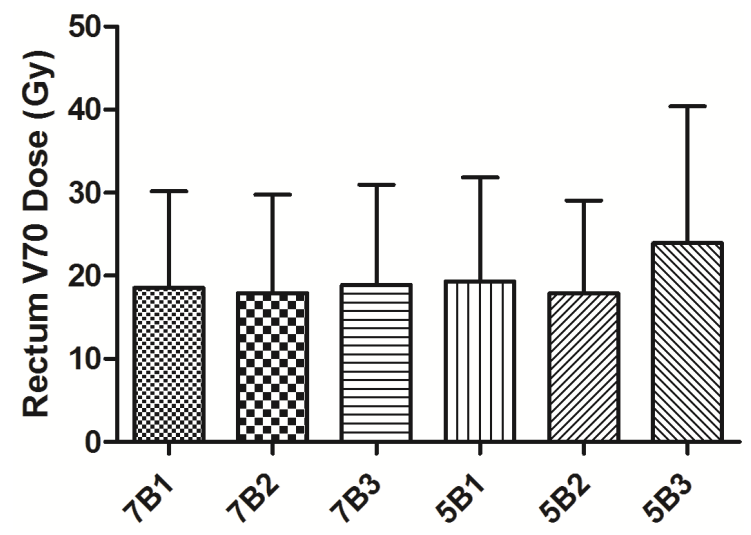

Figure 10. Rectum V70 dose for different IMRT plans.

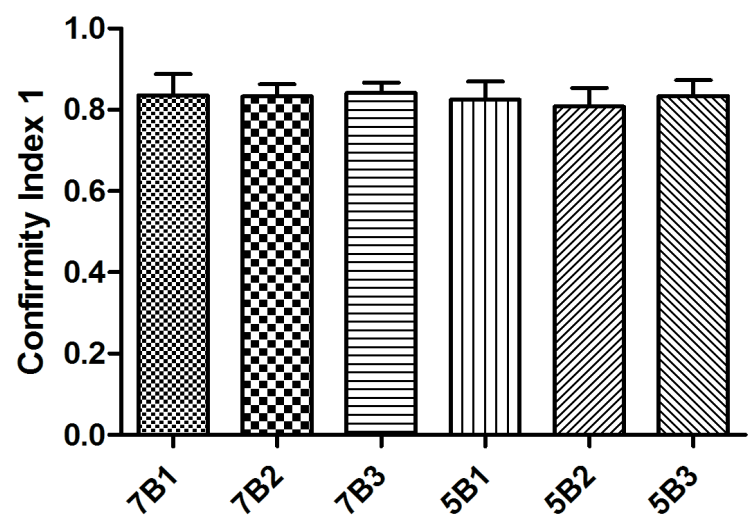

Figure 11. Conformity index of various IMRT plans.

MU of all 3 sets 668.288 Vs 817.387) as shown in Figure 13 and Table 15.

$>$ All IMRT plans, 3 different set of 7 beam gantry angles and 3 different set of 5 beam gantry angles, differed slightly in the measured parameters, however none of them was statistically significant.

Conformity index, Homogeneity index and monitor units all showed non-significant $(\mathrm{P}>0.05)$ difference between the various groups of different IMRT plans. 


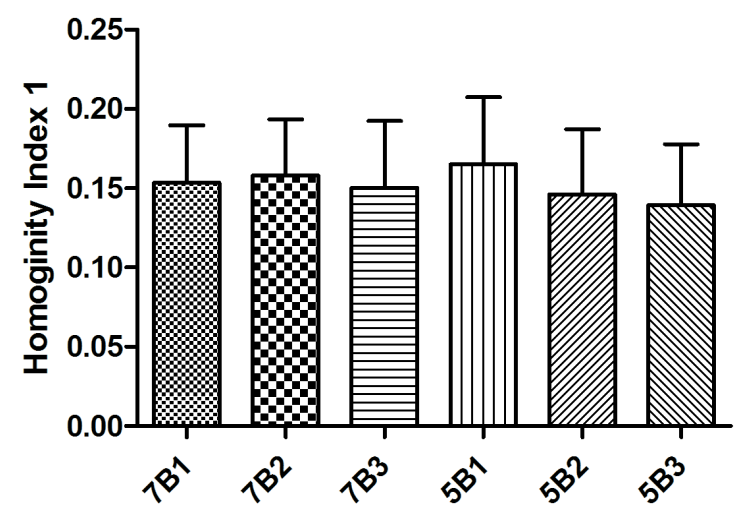

Figure 12. Homogeneity index of various IMRT plans.

\section{Monitor Unit/CC}

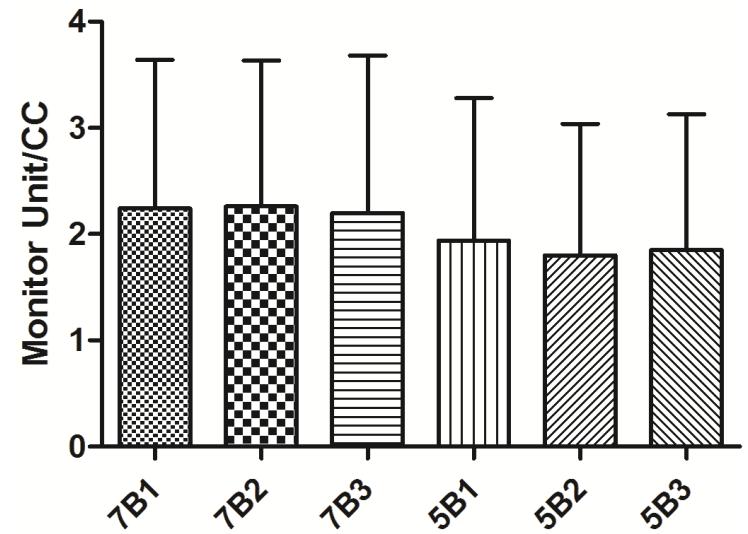

Figure 13. Monitor unit of various IMRT plans.

Table 9. Conformity index of various IMRT plans.

\begin{tabular}{ccc}
\hline & Conformity Index 1 & \\
\hline Group & Mean \pm SD & \\
\hline $7 \mathrm{~B} 1$ & $0.8357 \pm 0.0525$ & \\
$7 \mathrm{~B} 2$ & $0.8326 \pm 0.0307$ & $0.5242(\mathrm{~ns})$ \\
$7 \mathrm{~B} 3$ & $0.8411 \pm 0.0262$ & \\
$5 \mathrm{~B} 1$ & $0.8243 \pm 0.0454$ & \\
$5 \mathrm{~B} 2$ & $0.8075 \pm 0.0463$ & \\
$5 \mathrm{~B} 3$ & $0.8340 \pm 0.0392$ & \\
& &
\end{tabular}

However, 5 field IMRT plans achieved a 14.9\% relative decrease in the mean number of monitor units for radiation delivery as compared to 7 field IMRT plans.

\section{Discussion}

Prostate cancer is the most frequently diagnosed cancer among men in over 
Table 10. Tukey's multiple comparison test for conformity index of various IMRT plans.

\begin{tabular}{cc}
\hline $\begin{array}{c}\text { Tukey's Multiple Comparison Test, Significant (s), } \\
\text { Non-Significant (ns) Tukey's Multiple Comparison Test }\end{array}$ & Summary \\
\hline $7 \mathrm{~B} 1$ vs $7 \mathrm{~B} 2$ & $\mathrm{~ns}$ \\
$7 \mathrm{~B} 1$ vs $7 \mathrm{~B} 3$ & $\mathrm{~ns}$ \\
$7 \mathrm{~B} 1$ vs $5 \mathrm{~B} 1$ & $\mathrm{~ns}$ \\
$7 \mathrm{~B} 1$ vs $5 \mathrm{~B} 2$ & $\mathrm{~ns}$ \\
$7 \mathrm{~B} 1$ vs $5 \mathrm{~B} 3$ & $\mathrm{~ns}$ \\
$7 \mathrm{~B} 2$ vs $7 \mathrm{~B} 3$ & $\mathrm{~ns}$ \\
$7 \mathrm{~B} 2$ vs $5 \mathrm{~B} 1$ & $\mathrm{~ns}$ \\
$7 \mathrm{~B} 2$ vs $5 \mathrm{~B} 2$ & $\mathrm{~ns}$ \\
$7 \mathrm{~B} 2$ vs $5 \mathrm{~B} 3$ & $\mathrm{~ns}$ \\
$7 \mathrm{~B} 3$ vs $5 \mathrm{~B} 1$ & $\mathrm{~ns}$ \\
$7 \mathrm{~B} 3$ vs $5 \mathrm{~B} 2$ & $\mathrm{~ns}$ \\
$7 \mathrm{~B} 3$ vs $5 \mathrm{~B} 3$ & $\mathrm{~ns}$ \\
$5 \mathrm{~B} 1$ vs $5 \mathrm{~B} 2$ & $\mathrm{~ns}$ \\
$5 \mathrm{~B} 1$ vs $5 \mathrm{~B} 3$ & $\mathrm{~ns}$ \\
$5 \mathrm{~B} 2$ vs $5 \mathrm{~B} 3$ & \\
\hline
\end{tabular}

Significant (s), Non-Significant (ns).

Table 11. Homogeneity index of various IMRT plans.

\begin{tabular}{ccc}
\hline & Homogeneity Index 1 & \\
Group & Mean $\pm \mathrm{SD}$ & \\
\hline $7 \mathrm{~B} 1$ & $0.1535 \pm 0.0362$ & \\
$7 \mathrm{~B} 2$ & $0.1580 \pm 0.0354$ & $0.7527(\mathrm{~ns})$ \\
$7 \mathrm{~B} 3$ & $0.1504 \pm 0.0419$ & \\
5B1 & $0.1652 \pm 0.0423$ & \\
5B2 & $0.1462 \pm 0.0409$ & \\
5B3 & $0.1393 \pm 0.0385$ & \\
\hline
\end{tabular}

one-half of the countries of the world [10]. Several treatment alternatives exist for localized prostate cancer, including observation, prostatectomy, brachytherapy and external beam radiation therapy (EBRT). The outcome of the treatment of early prostate cancer has improved in recent years mainly due to the radiation dose escalation [11].

The major advantage of IMRT is the ability to decrease the dose to critical structures, which in turn lowers the radiation toxicity effects [12]. In recent years, dose escalation has been shown to improve the probability of local tumour 
Table 12. Tukey's multiple comparison test for homogeneity index of various IMRT plans.

\begin{tabular}{lc}
\hline Tukey's Multiple Comparison Test & Summary \\
\hline $7 \mathrm{~B} 1$ vs $7 \mathrm{~B} 2$ & $\mathrm{~ns}$ \\
$7 \mathrm{~B} 1$ vs $7 \mathrm{~B} 3$ & $\mathrm{~ns}$ \\
$7 \mathrm{~B} 1$ vs $5 \mathrm{~B} 1$ & $\mathrm{~ns}$ \\
$7 \mathrm{~B} 1$ vs $5 \mathrm{~B} 2$ & $\mathrm{~ns}$ \\
$7 \mathrm{~B} 1$ vs $5 \mathrm{~B} 3$ & $\mathrm{~ns}$ \\
$7 \mathrm{~B} 2$ vs $7 \mathrm{~B} 3$ & $\mathrm{~ns}$ \\
$7 \mathrm{~B} 2$ vs $5 \mathrm{~B} 1$ & $\mathrm{~ns}$ \\
$7 \mathrm{~B} 2$ vs $5 \mathrm{~B} 2$ & $\mathrm{~ns}$ \\
$7 \mathrm{~B} 2$ vs $5 \mathrm{~B} 3$ & $\mathrm{~ns}$ \\
$7 \mathrm{~B} 3$ vs $5 \mathrm{~B} 1$ & $\mathrm{~ns}$ \\
$7 \mathrm{~B} 3$ vs $5 \mathrm{~B} 2$ & $\mathrm{~ns}$ \\
$7 \mathrm{~B} 3$ vs $5 \mathrm{~B} 3$ & $\mathrm{~ns}$ \\
$5 \mathrm{~B} 1$ vs $5 \mathrm{~B} 2$ & $\mathrm{~ns}$ \\
$5 \mathrm{~B} 1$ vs $5 \mathrm{~B} 3$ & $\mathrm{~ns}$ \\
$5 \mathrm{~B} 2$ vs $5 \mathrm{~B} 3$ & \\
\hline
\end{tabular}

Significant (s), Non-Significant (ns).

Table 13. Monitor units of various IMRT plans.

\begin{tabular}{ccc}
\hline & Monitor Unit/CC & Kruskal-Wallis test (P value) \\
\hline Group & Mean \pm SD & \\
\hline $7 \mathrm{~B} 1$ & $2.2420 \pm 1.4000$ & \\
$7 \mathrm{~B} 2$ & $2.2630 \pm 1.3710$ & $0.4369(\mathrm{~ns})$ \\
$7 \mathrm{~B} 3$ & $2.1990 \pm 1.4820$ & \\
$5 \mathrm{~B} 1$ & $1.9380 \pm 1.3430$ & \\
$5 \mathrm{~B} 2$ & $1.8000 \pm 1.2370$ & \\
$5 \mathrm{~B} 3$ & $1.8530 \pm 1.2760$ & \\
\hline
\end{tabular}

control in prostate cancer [11] [13]. The increase of normal tissue integral dose with multiple beam radiation therapy during IMRT is also a major concern due to the potential risk of the secondary malignancies especially in younger patients [14].

IMRT uses beam modulation with the help of multileaf collimators and complicated inverse planning software and calculation algorithms. Beam configuration may have significant influence on the quality of an IMRT treatment. The planning process is time taking as it compares different beam configurations 
Table 14. Dunn's multiple comparison test of various IMRT plans.

\begin{tabular}{lc}
\hline Dunn's Multiple Comparison Test & Summary \\
\hline $7 \mathrm{~B} 1$ vs $7 \mathrm{~B} 2$ & $\mathrm{~ns}$ \\
$7 \mathrm{~B} 1$ vs $7 \mathrm{~B} 3$ & $\mathrm{~ns}$ \\
$7 \mathrm{~B} 1$ vs $5 \mathrm{~B} 1$ & $\mathrm{~ns}$ \\
$7 \mathrm{~B} 1$ vs $5 \mathrm{~B} 2$ & $\mathrm{~ns}$ \\
$7 \mathrm{~B} 1$ vs $5 \mathrm{~B} 3$ & $\mathrm{~ns}$ \\
$7 \mathrm{~B} 2$ vs $7 \mathrm{~B} 3$ & $\mathrm{~ns}$ \\
$7 \mathrm{~B} 2$ vs $5 \mathrm{~B} 1$ & $\mathrm{~ns}$ \\
$7 \mathrm{~B} 2$ vs $5 \mathrm{~B} 2$ & $\mathrm{~ns}$ \\
$7 \mathrm{~B} 2$ vs $5 \mathrm{~B} 3$ & $\mathrm{~ns}$ \\
$7 \mathrm{~B} 3$ vs $5 \mathrm{~B} 1$ & $\mathrm{~ns}$ \\
$7 \mathrm{~B} 3$ vs $5 \mathrm{~B} 2$ & $\mathrm{~ns}$ \\
$7 \mathrm{~B} 3$ vs $5 \mathrm{~B} 3$ & $\mathrm{~ns}$ \\
$5 \mathrm{~B} 1$ vs $5 \mathrm{~B} 2$ & $\mathrm{~ns}$ \\
$5 \mathrm{~B} 1$ vs $5 \mathrm{~B} 3$ & $\mathrm{~ns}$ \\
$5 \mathrm{~B} 2$ vs $5 \mathrm{~B} 3$ & \\
\hline
\end{tabular}

Significant (s), Non-Significant (ns).

Table 15. Comparison of the mean dosimetric parameters.

\begin{tabular}{|c|c|c|c|c|c|c|}
\hline \multirow{2}{*}{ Variable } & \multicolumn{3}{|c|}{$5 \mathrm{~B}$} & \multicolumn{3}{|c|}{$7 \mathrm{~B}$} \\
\hline & $5 \mathrm{~B} 1$ & $5 \mathrm{~B} 2$ & $5 \mathrm{~B} 3$ & $7 \mathrm{~B} 1$ & $7 \mathrm{~B} 2$ & 7B3 \\
\hline Monitor units & $694.787 \pm 134.8341$ & $646.965 \pm 123.1417$ & $663.113 \pm 131.6934$ & $832.63 \pm 160.8641$ & $832.907 \pm 149.6642$ & $786.625 \pm 149.4254$ \\
\hline $\begin{array}{l}\text { PTV volume of } 95 \% \\
\text { isodose }\left(\mathrm{cm}^{3}\right)\end{array}$ & $65.88 \pm 1.37$ & $66.48 \pm 1.34$ & $66.51 \pm 1.32$ & $66.37 \pm 1.26$ & $66.10 \pm 1.45$ & $66.45 \pm 1.27$ \\
\hline $\begin{array}{l}\text { Conformity index } \\
\text { (Volume of } \\
\text { 95\%/PTV) } 13\end{array}$ & $0.9671 \pm 0.09$ & $0.9692 \pm 0.08$ & $0.9814 \pm 0.07$ & $0.9787 \pm 0.07$ & $0.9676 \pm 0.09$ & $0.9757 \pm 0.07$ \\
\hline $\begin{array}{l}\text { Homogeneity index } \\
\text { (D5\%/D95\%) }\end{array}$ & $1.1161 \pm 0.02$ & $1.1023 \pm 0.02$ & $1.0986 \pm 0.02$ & $1.1063 \pm 0.02$ & $1.1073 \pm 0.02$ & $1.1044 \pm 0.02$ \\
\hline
\end{tabular}

from different angles to get the best optimization possible. This often becomes a tedious process, especially in centers with limited resources and large number of patients. Empirically, 5 to 7 field IMRT plans with various beam angles are used in treating prostate cancer. The use of an optimal starting gantry angle for 5 equiangular-spaced beams facilitate rectal sparing and can produce plans that are equivalent to those employing 7 equiangular-spaced beams [15]. Mahdavi et al. also concluded that in comparison to the 7 field technique, the 5 field IMRT technique has resulted in improved IMRT dose conformity, homogeneity, and lesser MUs used for radiation therapy [16]. 
In this study we have used 3 different sets of 5 beam gantry angle and 3 different sets of 7 beam gantry angle and tried to find the best combination which can be applied in general to our patients. The results show that though there are slight differences in various measured parameters, none of them are statistically significant. Both conformity index and Homogeneity index showed non-significant $(P>0.05)$ difference between the various groups of different IMRT plans. Monitor units also showed non-significance difference between various groups. However, 5 field IMRT plans achieved a $14.9 \%$ relative decrease in the mean number of monitor units for radiation delivery as compared to 7 field IMRT plans. Due to the lesser number of monitor units in 5 beam gantry angles, treatment was completed in lesser time and also resulted in lesser integral dose to normal tissue and lesser chance of secondary malignancy.

\section{Conclusion}

Radiation therapy for prostate cancer has evolved drastically over the past few years. The Intensity Modulated Radiation Therapy (IMRT) is a novel form of radiation delivery technique for prostate cancer which allows dose escalation and therefore improved tumour control. Various techniques in terms of number of beams and angles can be used to get the desired dose distribution, but the process is time-consuming. After trying 6 different combinations of beams in our patients we found that in terms of conformity index, homogeneity index and monitor units both 5 beam and 7 beam IMRT technique show non-significant difference. However, 5 field IMRT plans use a lesser number of monitor units per treatment than 7 field IMRT plans. A decrease in monitor units also leads to the lesser linear accelerator beam-on time and consequently lesser treatment time and running cost. Thus, in a high-volume centre with 5 field technique we can treat more patients in lesser time.

\section{Limitation of This Study}

1) Small sample size.

2) The clinical correlation of doses received by OARs in this of acute \& late toxicities was not done.

3) Require prospective, randomized study with large sample size and long term follow up for assessing significant clinical outcome.

\section{Ethical Clearance}

The study is a comparison of different treatment plans in the same data set. However, there was no change in the patient's treatment plan for the proposed study. So ethical committee clearance was not required.

The study has been approved and allowed by the Head of the Institute.

\section{Acknowledgements}

Dr. Richa Chauhan, MCS, Patna. 


\section{Conflicts of Interest}

None.

\section{Finical Support}

None.

\section{References}

[1] Hanks, G.E., Hanlon, A.L., Epstein, B., et al. (2002) Dose Response in Prostate Cancer with 8-12 Years' Follow-Up. International Journal of Radiation Oncology, Biology, Physics, 54, 427-435. https://doi.org/10.1016/S0360-3016(02)02954-1

[2] Dearnaley, D.P., Sydes, M.R., Graham, J.D., et al. (2007) Escalated-Dose versus Standard-Dose Conformal Radiotherapy in Prostate Cancer: First Results from the MRC RT01 Randomised Controlled Trial. The Lancet Oncology, 8, 475-487. https://doi.org/10.1016/S1470-2045(07)70143-2

[3] Peeters, S.T., Heemsbergen, W.D., Koper, P.C., et al. (2006) Dose-Response in Radiotherapy for Localized Prostate Cancer: Results of the Dutch Multicenter Randomized Phase III Trial Comparing 68 Gy of Radiotherapy with 78 Gy. Journal of Clinical Oncology, 24, 1990-1996. https://doi.org/10.1200/JCO.2005.05.2530

[4] Zelefsky, M.J., Chan, H., Hunt, M., et al. (2006) Long-Term Outcome of High Dose Intensity Modulated Radiation Therapy for Patient with Clinically Localized Prostate Cancer. Journal of Urology, 176, 1415-1419.

https://doi.org/10.1016/j.juro.2006.06.002

[5] Shu, H.K., Lee, T.T., Vigneauly, E., et al. (2001) Toxicity Following High-Dose Three-Dimensional Conformal and Intensity-Modulated Radiation Therapy for Clinically Localized Prostate Cancer. Urology, 57, 102-107. https://doi.org/10.1016/S0090-4295(00)00890-6

[6] Stein, J., Mohan, R., Wang, X.H., et al. (1997) Number and Orientations of Beams in Intensity-Modulated Radiation Treatments. Medical Physics, 24, 149-160. https://doi.org/10.1118/1.597923

[7] Schreibmann, E. and Xing, L. (2004) Feasibility Study of Beam Orientation ClassSolutions for Prostate IMRT. Medical Physics, 31, 2863-2870. https://doi.org/10.1118/1.1797571

[8] Semerenko, V.A., Reitz, B., Day, E., Qi, X.S., Miften, M. and Li, X.A. (2008) Evaluation of a Commercial Biologically Based IMRT Treatment Planning System. Medical Physics, 35, 5851-5860. https://doi.org/10.1118/1.3013556

[9] Shaw, E., Kline, R., Gillin, M., Souhami, L., Hirschfeld, A., Dinapoli, R., et al. (1993) Radiation Therapy Oncology Group: Radiosurgery Quality Assurance Guidelines. International Journal of Radiation Oncology, Biology, Physics, 27, 1231-1239. https://doi.org/10.1016/0360-3016(93)90548-A

[10] Bray, F., et al. (2018) Global Cancer Statistics 2018: GLOBOCON Estimates of Incidence and Mortality Worldwide for 36 Cancers in 185 Countries. CA: A Cancer Journal for Clinicians, 68, 394-424. https://doi.org/10.3322/caac.21492

[11] Al-Mamgani, A., van Putten, W.L., Heemsbergem, W.D., et al. (2008) Update of Dutch Multicentre Dose-Escalation Trial of Radiotherapy for Localized Prostate Cancer. International Journal of Radiation Oncology, Biology, Physics, 72, 980-988. https://doi.org/10.1016/j.ijrobp.2008.02.073

[12] Michalski, J.M., Yan, Y., Watkins-Bruner, D., et al. (2013) Preliminary Toxicity 
Analysis of 3DCRT versus IMRT on the High Dose Arm of the RTOG 0126 Prostate Cancer Trial. International Journal of Radiation Oncology, Biology, Physics, 87, 932-938. https://doi.org/10.1016/j.ijrobp.2011.06.004

[13] Zelefsy, M.J., Cowen, D., Fuks, Z., et al. (1999) Long Term Tolerance of High Dose Three-Dimensional Conformal Radiotherapy in Patients with Localized Prostate Cancer. Cancer, 85, 2460-2468.

https://doi.org/10.1002/(SICI)1097-0142(19990601)85:11<2460::AID-CNCR23>3.0. CO;2-N

[14] Hall, E.J. and Wu, C.S. (2003) Radiation-Induced Second Cancers: The Impact of 3D-CRT and IMRT. International Journal of Radiation Oncology, Biology, Physics, 56, 83-88. https://doi.org/10.1016/S0360-3016(03)00073-7

[15] Potrebko, P.S., McCurdy, B.M., Butler, J.B., et al. (2007) Optimal Starting Gantry Angles Using Equiangular-Spaced Beams with Intensity Modulated Radiation Therapy for Prostate Cancer on RTOG 0126: A Clinical Study of 5 and 7 Fields. Radiotherapy and Oncology, 85, 299-305. https://doi.org/10.1016/j.radonc.2007.06.019

[16] Mahdavi, S., JazayeriGharehbagh, E., Nikoofar, A., et al. (2017) Radiation Treatment Planning for Prostate Cancer: A New Dosimetric Comparison of Five and Seven Fields IMRT Plans. International Journal of Radiation Research, 15, 177-183. https://doi.org/10.18869/acadpub.ijrr.15.2.177 